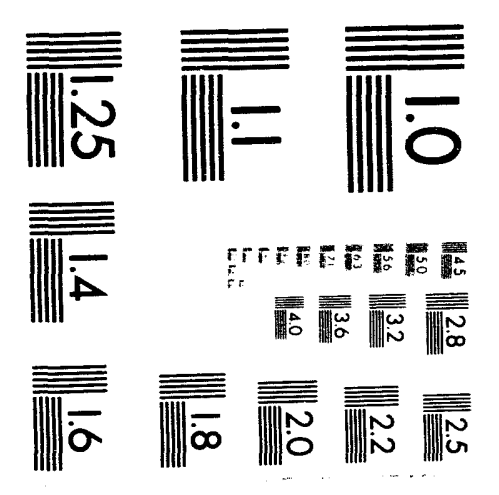



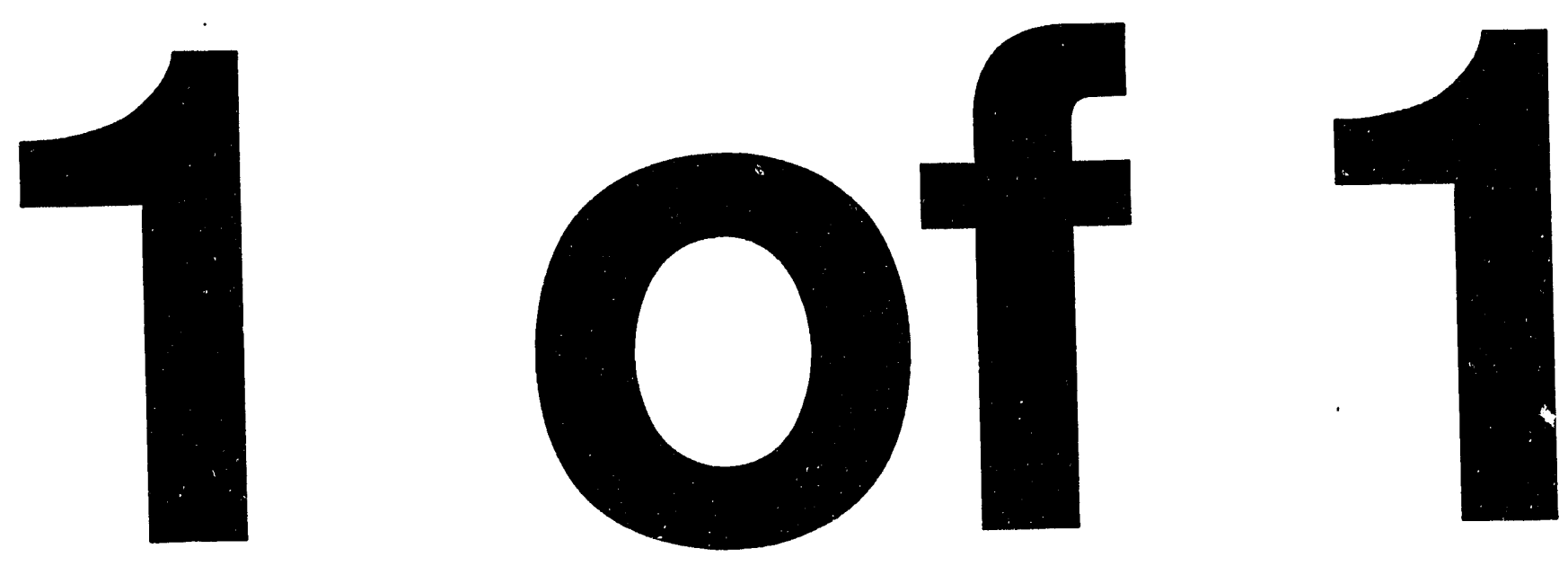
Final Report to the United States Department of Energy on Research Supported in Part Under Contract DE-AS05-80ER-10666

\title{
ADVANCED METHODS FOR THE COMPUTATION OF PARTICLE BEAM TRANSPORT AND THE COMPUTATION OF ELECTROMAGNETIC FIELDS AND BEAM-CAVITY INTERACTIONS
}

\author{
Alex J. Dragt \\ Robert L. Gluckstern \\ Department of Physics \\ University of Maryland \\ College Park, Maryland 20742
}

November 1992

\section{DISCLAIMER}

This report was prepared as an account of work sponsored by an agency of the United States Government. Neither the United States Government nor any agency thereof, nor any of their employees, makes any warranty, express or implied, or assumes any legal liability or responsi. bility for the accuracy, completeness, or usefulness of any information, apparatus, product, or process disclosed, or represents that its use would not infringe privately owned rights. Reference herein to any specific commercial product, process, or service by trade name, trademark, manufacturer, or otherwise does not necessarily constitute or imply its endorsement, recommendation, or favoring by the United States Government or any agency tiereof. The views and opinions of authors expressed herein do not necessarily state or reflect those of the United States Government or any agency thereof.

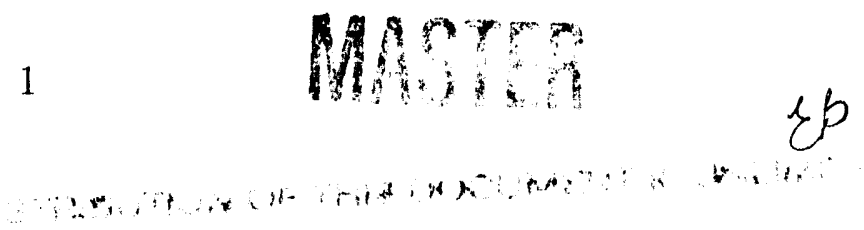




\section{ABSTRACT}

The University of Maryland Dynamical Systems and Accelerator Theory Croup carries out research in two broad areas: the computation of charged particle beam transport using Lie algebraic methods and advanced methods for the computation of electromagnetic fields and beam-cavity interactions. Important improvements in the state of the art are believed to be possible in both of these areas. In addition, applications of these methods are made to problems of current interest in accelerator physics including the theoretical performance of present and proposed high energy machines.

The Lie algebraic method of computing and analyzing beam transport handles both linear and nonlinear beam elements. Tests show this method to be superior to the earlier matrix or numerical integration methods. It has wide application to many areas including accelerator physics, intense particle beams, ion microprobes, high resolution electron microscopy, and light optics.

With regard to the area of electromagnetic fields and beam cavity interactions, work is carried out on the theory of beam breakup in single pulses. Work is also done on the analysis of the high frequency behavior of longitudinal and transverse coupling impedances, including the examination of methods which may be used to measure these impedances. Finally, work is performed on the electromagnetic analysis of coupled cavities and on the coupling of cavities to waveguides.

In addition to its research effort, the Dynamical Systems and Accelerator Theory Group is actively engaged in the education of students and postdoctoral research associates. To this end, it presents a regular graduate seminar course in accelerator physics, directs graduate students in M.S. and Ph.D. thesis research, and guides and fosters the research of post-doctoral research associates.

Substantial progress in research has been made during the past contract/grant period. There have also been important educational accomplishments. These achievements are summarized in the following report. 


\section{Educational Accomplishments}

One of the major goals of the Dynamical Systems and Accelerator Theory Group is the education of students, postdoctoral research associates, and visiting scientists. Listed below are present and past students associated with the program, postdoctoral research associates, and visiting scientists.

\subsection{Present Graduate Students}

Dan Abell

Thesis work in progress on the computation and analytic properties of transfer maps.

Wen Hao Cheng

Now in his second year as a Graduate Research Assistant studying the current carrying capacity of an alternating phase focussing linac. He is also starting to work on the calculation of the transverse impedance of an obstacle in a beam pipe.

Shicheng Jiang

Now in his first year as a Graduate Research Assistant working on the impedance of a thick iris.

\subsection{Past Graduate Students}

David Douglas, Ph.D. 1982.

Thesis: Lie Algebraic Methods for Particle Accelerator Theory.

Employment: Previously employed by the Lawrence Berkeley Laboratory. Presently employed by the Continuous Electron Beam Accelerator Facility.

Etienne Forest, Ph.D. 1984.

Thesis: Lie Algcbraic Methods for Charged Particle Beams and Light Optics.

Employment: Currently employed by the Lawrence Berkeley Laboratory. 
Janko Milutinovic, Ph.D. 1984.

Thesis work done in Elementary Particle Theory while simultaneously receiving training in Accelerator Theory.

Employment: Accelerator Theory at Brookhaven National Laboratory.

Liam Healy, Ph.D. 1986.

Thesis: Lie Algebraic Methods for Treating Lattice Parameter Errors in Particle Accelerators.

Employment: Previously at CERN. Presently with celestial mechanics group at the Naval Research Laboratory.

Robert Ryne, Ph.D. 1987.

Thesis: Lie Algebraic Treatment of Space Charge.

Employment: Previously employed by Lawrence Livermore National Laboratory. Presently employed by the Los Alamos National Laboratory.

Petra Schuett (1987-88).

Worked one year at Maryland on Lie algebraic methods and Poisson solvers while simultaneously completing her Ph.D. thesis work on Wake-Field Acceleration under Thomas Weiland at DESY.

Employment: Presently with the Theoretische Elektrotechnik Group at the Darmstadt Technische Hochschule.

Govindan Rangarajan, Ph.D. 1990.

Thesis: Invariants for Symplectic Maps and Symplectic Completion of Symplectic Jets.

Employment: Lawrence Berkeley Laboratory.

Rui Li, Ph.D. 1990.

Thesis: Analytic and Numerical Investigation of the Longitudinal Coupling Impedance

Employment: Continuous Electron Beam Accelerator Facility. 
John Diamond, M.S. 1990

Spent two years as a Graduate Research Assistant working on the penetration of electromagnetic fields through circular holes in a thick metallic wall. Also carried out symbolic manipulation program calculations for various complicated beamline elements such as combined function quadrupoles and combined function bends. In addition, responsible for general computational support. Now working at Adroit Systems, Alexandria, VA.

William Detlefs, M.S. (in Applied Math with Thesis) 1991

Thesis: Longitudinal Coupling Impedance of a Thin Iris Collimator.

Employment: Now a graduate student with support in Physics at Cal Tech.

\subsection{Other Students}

Dimitris Vassiliadis (1987-88)

Spent $11 / 2$ years working as a Graduate Research Assistant on Alternating Phase Focussing. Now affiliated with a group working on chaos.

Jeffrey Skibo (1988-89)

Spent one year exploring possible further work on the use of Lie algebraic methods in the treatment of space charge. Also responsible for general computational support. Now working in space physics.

Eric Salter (1990-91)

Spent one year working on the calculation of the impedance of a thick iris. Also did programming for MARYLIE and provided general computational support. Now serving as a Teaching Assistant.

Glenn VanderWoude (1988- )

Undergraduate student and National Merit Scholar. Responsible for general computational support. 


\subsection{Present Ph.D. Professional Staff}

Fernando Casas (1992-)

Visiting postdoctoral fellow, supported by Spanish Government.

Ivan Gjaja (1991-)

Postdoctoral research associate and SSC fellow.

Branislav Radak (1992-)

Postdoctoral research associate.

Johannes van Zeijts (1987-)

Postdoctoral research associate.

\subsection{Past Ph.D. Professional Staff}

Herbert O. Moser (1985)

Visiting Scientist. Presently at IMM Institut fur Mikrotechnik, Mainz Germany.

Hiromi Okamoto (1989-90)

Visiting postdoctoral research associate. Presently at Nuclear Science Laboratory, Institute for Chemical Research, Kyoto Japan.

Filippo Neri (1983-90)

Postdoctoral Research Associate. Presently at Los Alamos National Laboratory.

\section{Research Accomplishments}

Most of the major objectives set forth in the 1989 proposal have been met, and additional objectives have been achieved as well. A brief summary of work accomplished over the last contract/grant period is given below. 


\subsection{Task A: Charged Particle Beam Transport}

1. The program MARYLIE 3.0, comprising approximately 32,000 lines of code, is nearly ready for wide distribution and general use. A detailed instruction manual, consisting of some 470 pages and set in TEX, is substantially complete. ${ }^{1}$ Preliminary versions of the code have been tested on VAX, CRAY, IBM, SUN, and UNIX computers, and are in use in various laboratories in the United States, Canada, and Europe.

2. Several major improvements and additions have been made to various MARYLIE components. Some of these improvements and additions are listed below:

(a) The MARYLIE fitting routines have received dramatic improvements. Suppose the device or machine under study has a set of properties $A$ (focal lengths, aberrations, tunes, chromaticites, etc.), and we wish to adjust various parameters to achieve a set of properties $B$ that may be quite far from $A$. A new MARYLIE procedure determines a set of target values that moves continuously from $A$ to $B$. The MARYLIE fitting routines then continously adjust parameter values to follow the moving target values until the final target $B$ values are achieved. This new algorithm has remarkable convergence properties, and allows the solution of fitting problems that were previously intractable or impossible by earlier techniques.

(b) Optimizing routines have been added. These routines adjust parameters to optimize arbitrary merit functions, and are of use when fits are not possible.

(c) Scanning routines have been added that facilitate parameter studies. These routines automatically scan parameter space, and prepare convenient graphical output.

(d) The logic of the fitting, optimizing, and scanning procedures has been extended so that it is possible to fit in an "inner loop" while scanning or optimizing in an "outer loop".

(e) A routine has been added to MARYLIE for the numerical computation of the transfer map for a combined function bend. ${ }^{2} \mathrm{~A}$ 
combined function bend is a sector dipole with superimposed normal and skew quadrupole, sextupole, and octupole fields. This MARYLIE feature has been used extensively at Brookhaven National Laboratory for the design of compact synchrotron light sources with superconducting bends.

(f) A routine has been added to MARYLIE for the analytical computation of the transfer map for a combined function quadrupole. ${ }^{3} \mathrm{~A}$ combined function quadrupole is a quadrupole with superimposed normal and skew sextupole and octupole fields. The FORTRAN for this routine was produced by symbolic manipulation, and comprises some 3000 lines of dense code. This new MARYLIE feature, because of its high execution speed, has been essential to the design and ultimate success of the final expansion telescope for the Los Alamos Neutral Particle Beam program.

(g) A routine has been added to MARYLIE for the numerical computation of the transfer map for a solenoid including fringe-ficld effects. "The addition of this routine makes it possible to model beam transport systems with solenoids. The effect of solenoidal fields in detectors and the skew quadrupole correction of solenoidal effects can also be studied. Finally, the performance of electron microscopes can be studied.

(h) Routines have been added to MARYLIF for the generation of various particle distributions. 'The generation and subsequent tracking of these distributions is useful for the study of the performance of transport lines, telescopes, and final focus systems. 'These routines also calculate all moments, through order 6 , of the various distributions. These moments are of interest for studying emittance growth.

3. A version of MARYIIE 3.1, a third-order code including error effects, has been assembled and used to study the importance of misalignment effects in the Final Focus Test Beam facility under construction at Stanford."

4. Versions of MARYLIE 5.0, a fifth-order code, are in use at Los Alamos. These versions contain extensive (iENMAP routines that compute trans- 
fer maps for octupole and decupole corrected rare-earth-cobalt quadrupoles including all fringe field effects. ${ }^{6,7,8}$

5. Work has begun on MARYLIE 5.1, a fifth-order code including error effects.

6. Lie algebraic map multiplication rules have recently been worked out through seventh order using symbolic manipulation. These rules are a key step in the construction of a seventh-order Lie algebraic program.

7. A new hybrid code that uses both Taylor series and Lie algebraic methods has been developed. This code, called Tlie, works numerically to arbitrary order. ${ }^{9}$ That is, given any Hamiltonian expanded to some order, it can compute by numerical means the associated transfer map to the same order. It can also multiply maps of any specified order. Tlie has been used to cross check other existing codes, and for fifth and higher order aberration studies at Los Alamos. In addition, it has been used to study the importance of misalignment and higher-order aberration effects for the Stanford Final Focus Test Beam Facility. ${ }^{5}$

8. The computation of error effects in nonlinear Hamiltonian systems using Lie algebraic methods has been worked out in detail, and applied to the problem of steering magnets and mispowered dipoles. ${ }^{10}$ These computations will be implemented in MARYLIE 3.1 and 5.1, and cross checked with Tlie.

9. Self consistent Lie algebraic calculations have been carried out for cylindrically symmetric beams with linear and nonlinear space-charge effects. ${ }^{11}$ These calculations agree well with particle in cell simalations. However, they are orders of magnitude faster since they compute the transfer map directly rather than follow the trajectories of a large number of particles. Moreover, they provide aberration data that is inaccessible to particle in cell codes due to their large statistical noise.

10. A theory and procedure has been developed for the construction of complete third-order achromats. ${ }^{12}$ A complete third-order achromat bends a charged particle beam, but otherwise has an identity transfer map through third order for the full six-dimensional phase space including 
time of flight terms. The theory of such achromats makes use of normal form procedures. It is expected that such achromats may be of use in the design of time of flight mass spectrometers and certain high performance storage rings.

11. General moment invariants have been found for linear Hamiltonian systems. ${ }^{13,14,15}$ This work generalizes the concept of emittance to the full six-dimensional phase space, and shows that there are three conserved eigen emittances (constructed from second-order moments) for general linear Hamiltonian systems. It also shows that there are an infinite number of invariants for such systems that can be constructed from third and higher-order moments. These higher-order invariants may be of use for studying nonlinear effects.

12. Based on the work just described, a general new theory has been developed for the description of beams in a full six-dimensional phase space. ${ }^{16}$ This theory generalizes the usual Twiss beam envelope description (used for the earlier two-dimensional phase space description) by the introduction of envelope matrices and eigen phase advances in addition to the eigen emittances described above. The computation of eigen emittances, envelope matrices, and eigen phase advances has been made a standard MARYLIE command. It is expected that these new tools will facilitate the calculation and description of projected emittance growth due to linear cross couplings. ${ }^{17}$

13. Work has begun on the computation of the dynamic aperture for a ring using the one-turn map for tracking studies. ${ }^{18}$ This method appears promising provided suitable procedures can be found for symplectifying high-order Taylor expansions. If successful, tracking using the one-turn map (or at least a small number of lumps) should be orders of magnitude faster than the usual element to element tracking, and should also permit a much more accurate treatment of real magnets with distributed multipoles and fringe-field effects.

14. The use of Taylor series maps raises at least two questions. First, what is the domain of convergence of the series? Second, how can a truncated expansion be made symplectic? Work has begun on the first 
question as part of the thesis research of Dan Abell, and preliminary results have been found for the anharmonic oscillator and the pendulum problem. Work on the second question constituted part of the thesis of Dr. Govindan Rangarajan, and more work on this question is in progress. ${ }^{15,19,20}$

15. Some of the work undertaken, both on symplectification methods and the description of charged particle beam transport using moments, raises various questions concerning the symplectic group $S p(6)$ and the full group of symplectic maps. Some of these questions have been answered including certain questions about $S p(6)$ representations and questions about the existence of invariant metrics. ${ }^{15,21}$ It is expected that these tools will be of use for further study. For example, representation theory is useful in the construction and understanding of invariants such as emittance invariants. Invariant metrics may be useful in characterizing the nonlinear component of a transfer map, and the convergence of various symplectification procedures.

16. Work has begun on writing an extensive set of Lecture Notes on Nonlinear Dynamics and Lie Methods with Applications to Accelerator Physics. ${ }^{22}$

\subsection{Task B: Computation of Electromagnetic Fields and Beam-Cavity Interactions}

1. Beam Cavity Interactions/Beam Breakup

Work was completed on the theory of beam breakup with coupling between cavities [a]. Results were obtained as a function of the ratio of $Q^{-1}$ to the dispersion curve band width and to the separation of modes. For zero band width the results reduce to those for cumulative beam breakup. For $Q^{-1}$ less than the separation between the modes, the results reduce to regenerative beam breakup. Thus our formalism contains the more general case, but reduces to the usual results in these limits.

The theory of single bunch beam breakup has been extended to include BNS damping as well as Autophasing $[b, c, d]$. Universal curves have 
been obtained for the suppression of beam breakup as a function of the energy gradient introduced into the bunch and these have been compared with the formalism of Balakin [e].

\section{Coupling Impedance}

The coupling impedance of an obstacle in a beam pipe was formulated in terms of an integral equation for the axial electric field at the wall at the location of the obstacle [f]. This formulation permitted the study of the impedance in the high frequency limit [f], as well as for a periodic array of obstacles [g]. In addition results were obtained for a small obstacle [h] as well as for a large number of obstacles [i] in order to study the transition to the periodic result. In this work confirmation was obtained of the prediction by Palmer [j] that the impedance would increase only as the square root of the number of obstacles due to a "shadowing" effect. The integral equation method was also applied to the calculation of the transmission coefficient for a thin wire in the same cavity geometry $[\mathrm{k}]$, and the results showed that the wire measurement of impedance is more reliable than had been anticipated. All of these calculations are described in detail in Rui Li's thesis [ $\ell]$. In addition, the method was also applied to the calculation of the impedance of a multi-cell, multi-block structure $[\mathrm{m}]$.

3. Impedance of an Iris Collimator

A method has been developed to calculate the impedance of an iris within a beam pipe $[n]$. Numerical calculations have been carried out for zero iris thickness [o] and asymptotic results obtained for a small iris in both the low and high frequency limits.

4. Penetration of Fields through Holes in a Conducting Wall

Work has been completed on the calculation of the penetration of electromagnetic fields through small holes of general shape in a wall of zero thickness [p]. In this analysis, variational forms are constructed for the polarizability and susceptibilities of the hole. With judiciously chosen trial functions, one can obtain accurate values of these parameters for a variety of shapes. The procedure was then extended to a circular hole in a thick wall $[q]$ and numerical values accurate to at least 4 significant figures were obtained over the complete range of the ratio wall 
thickness/hole radius from 0 to $\infty$.

5. Free Electron Lasers

A universal scaling function has been obtained for FEL gain for a laser including optical guiding [ $\mathrm{r}$. This is obtained from a variational formulation in the exponential region before saturation, incorporating the energy spread, emittance, and focussing of the electron beam and the diffraction and guiding of the radiation.

The fields in a variety of compact wiggler designs have been calculated. These included a bifilar helix [s] and a slotted cylindrical shell $[\mathrm{t}]$.

The effect of wiggler errors on FEL gain has also been calculated [u] with results that are somewhat less serious than earlier estimated.

6. Miscellaneous

During the contract period, a number of other reports have been written primarily at LANL and CERN on a variety of subjects.

\section{REFERENCES}

\subsection{Task A: Charged Particle Beam Transport}

1. A.J. Dragt et al., MARYLIE 3.0 User's Manual, A Program for Charged Particle Beam Transport Based cn Lie Algebraic Methods (1991).

2. A.J. Dragt, F. Neri, et al., Numerical Third-Order Transfer Map for Combined-Function Dipole, to be submitted to Nuclear Instruments and Methods (1990).

3. J. van Zeijts, F. Neri, et al., Analytic Third-Order Transfer Map for Combined-Function Quadrupole, to be submitted to Nuclear Instruments and Methods (1990).

4. A.J. Dragt, Numerical Third-Order Transfer Map for Solenoid, Nuclear Instruments and Methods A298, p. 441 (1990).

5. A.J. Dragt, Review of the SLAC Final Focus Test Beam Facility (1990). 
6. P. Walstrom, F. Neri, and T. Mottershead, High Order Optics of Multipole Magnets, LANL Report (1990).

7. P. Walstrom, Magnetic Fields and Inductances of Cylindrical Current Sheet Magnets, LANL Report (1990).

8. P. Walstrom, Magnetic Fields from Distributions of Dipoles on Cylindrical Surfaces, LANL Report (1991).

9. J. van Zeijts and F. Neri, The Arbitrary Order Design Code Tlie 1.0 (1992).

10. L. Healy, A.J. Dragt, and I. Gjaja, Computation of Error Effects in Nonlinear Hamiltonian Systems Using Lie Algebraic Methods, to appear in Journal of Mathematical Physics (1992).

11. R. Ryne and A.J. Dragt, Magnetic Optics Calculations for Cylindrically Symmetric Beams with Space Charge, Particle Accelerators 35, p. 129 (1991).

12. A.J. Dragt, Theory of Complete Third-Order Achromat, invited talk to be presented at Spring 1992 American Physical Society meeting.

13. F. Neri and G. Rangarajan, Kinematic Moment Invariants for Linear Hamiltonian Systems, Phys. Rev. Lett. 64, p. 1073 (1990).

14. A.J. Dragt et al., General Moment Invariants for Linear Hamiltonian Systems, Physical Review A, 45 (1992).

15. G. Rangarajan, Invariants for Symplectic Maps and Symplectic Completion of Symplectic Jets, U. of Maryland Ph.D. thesis (1990).

16. A.J. Dragt, Envelope Matrix Description of Linear Beam Transport, manuscript in preparation (1992).

17. K.L. Brown and R.V. Servranckx, Cross-Plane Coupling and Its Effect on Projected Emittance, Particle Accelerators 36, p. 121 (1991).

18. Y. Yan, A.J. Dragt, et al., Comment on Round-Off Errors and On OneTurn Taylor Maps, Nonlinear Problems in Future Particle Accelerators, W. Scandale and G. Turchetti, Ed., World Scientific (1991). 
19. A.J. Dragt, I.M. Gjaja and G. Rangarajan, Kick Factorization of Symplectic Maps, Proceedings of the IEEE Accelerator Conference, p. 1621 (1991).

20. I. Gjaja, Exact Evaluation of Arbitrary Symplectic Maps (1992).

21. A.J. Dragt, G. Rangarajan, and F. Neri, Invariant Metrics for Hamiltonian Systems, Proceedings of the IEEE Accelerator Conference, p. 1630 (1991).

22. A.J. Dragt, Lectures on Nonlinear Dynamics and Lie Methods with Applications to Accelerator Physics (225 pp.)(1991).

\subsection{Task B: Computation of Electromagnetic Fields and Beam-Cavity Interactions}

[a] R.L. Gluckstern and F. Neri, Beam Breakup with Coupling Between Cavities, Particle Accelerators, 25, 11 (1989).

[b] F. Neri and R.L. Gluckstern, Single Bunch Cumulative Beam Breakup, Proceeding of the Particle Accelerator Conference, Chicago, IL, March 1989, p. 812 .

[c] R.L. Gluckstern, F. Neri and J.B.J van Zeijts, Suppression of Single Bunch Beam Breakup by BNS Damping, Proceedings of the Linac Conference, Albuquerque, NM (1990), p. 309.

[d] R.L. Gluckstern and J.B.J. van Zeijts, Suppression of Single Bunch Beam Breakup by Autophasing, Proceedings of the Particle Accelerator Conference, San Francisco, CA (1991), p. 452.

[e] V.E. Balakin, Proceedings of the 1988 Workshop on Linear Colliders, SLAC, p. 55.

[f] R.L. Gluckstern, High Frequency Behavior of the Longitudinal Impedance for a Cavity of General Shape, Phys. Rev. D, 39, 2773 (1989). 
[g] R.L. Gluckstern, Longitudinal Impedance of a Periodic Structure at High Frequency, Phys. Rev. D, $\underline{39}, 2780$ (1989).

[h] R.L. Gluckstern and F. Neri, Longitudinal Coupling Impedance of a Small Obstacle, Proceedings of the Particle Accelerator Conference, Chicago, IL, March 1989, p. 1271.

[i] R.L. Gluckstern, High Frequency Dependence of the Coupling Impedance for a Large Number of Obstacles, Proceedings of the Particle Accelerator Conference, Chicago, IL, March 1989, p. 1157.

[j] R.B. Palmer, A Qualitative Study of Wakefields for Very Short Bunches, SLAC Report SLAC-PUB-4433, October 1987.

[k] R.L. Gluckstern and R. Li, Analysis of Coaxial Wire Measurement of Longitudinal Coupling Impedance, Particle Accelerators, 299, 159 (1990).

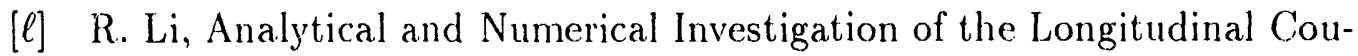
pling Impedance, Ph.D. thesis, University of Maryland, 1990.

[m] I. Gjaja, R. Li and R.L. Gluckstern, Impedance for a Multi-Cell, MultiBlock Structure, Proceedings of the Particle Accelerator Conference, San Francisco, CA, May 1991, p. 452.

[n] R.L. Gluckstern, Longitudinal Coupling Impedance of an Iris in a Beam Pipe, CERN Report SL/90-113 (AP) (1990).

[o] R.L. Gluckstern and W.F. Detlefs, Longitudinal Coupling Impedance of a Thin Iris Collimator, Proceedings of the Particle Accelerator Conference, San Francisco, CA, May 1991, p. 1600.

[p] R.L. Gluckstern, R. Li and R.K. Cooper, Electric Polarizability and Magnetic Susceptibility of Small Holes in a Thin Screen, IEEE Transactions in Microwave Theory and Techniques Vol. 38, No. 2, 186 (1990); see also Vol. 38, No. 10 (1990), pp.1529-1530. 
$[q]$ R.L. Gluckstern and J.A. Diamond, Penetration of Fields through a Circular Hole in a Wall of Finite Thickness, IEEE Transactions in Microwave Theory and Techniques, Vol. 39, No. 2, 274 (1991).

[r] L.H. Yu, S. Krinsky and R.L. Gluckstern, Calculation of a Universal Scaling Function for Free Electron Laser Gain, Phys. Rev. Letters 64, 3011 (1990).

[s] R.L. Gluckstern, Orbit Dynamics in the Field of a Bifilar Helix, LANL Report AT-6:ATN-89-20 (1989); Effects of Fringe Fields and Errors in a Bifilar Helix, AT-6:ATN-89-21 (1989).

[t] R.L. Gluckstern, Slotted Cylindrical Shell for Use as a Compact Wiggler, Phys. Rev. A15, 44, 3889 (1991).

[u] L.H. Yu, S. Krinsky, R.L. Gluckstern and J.B.J. van Zeijts, The Effects of Wiggler Errors on FEL Gain, Phys. Rev. A15, ㄴ5, 1163 (1992).

\section{Publications and Reports Written During Contract Period}

\subsection{Task A}

A.J. Dragt, F. Neri, et al., Numerical Third-Order Transfer Map for CombinedFunction Dipole, to be submitted to Nuclear Instruments and Methods (1990).

J. van Zeijts, F. Neri, et al., Analytic Third-Order Transfer Map For CombinedFunction Quadrupole, to be submitted to Nuclear Instruments and Methods (1990).

A.J. Dragt, Numerical Third-Order Transfer Map for Solenoid, Nuclear Instruments and Methods A298, p. 441 (1990). 
R. Ryne and A.J. Dragt, Magnetic Optics Calculations For Cylindrically Symmetric Beams with Space Charge, Particle Accelerators 35, p. 129 (1991).

L. Healy, A.J. Dragt, and I. Gjaja, Computation of Error Effects in Nonlinear Hamiltonian Systems Using Lie Algebraic Methods, to appear in Journal of Mathematical Physics (1992).

A.J. Dragt, R.L. Gluckstern, et al., Theory of Emittance Invariants, Lecture Notes in Physics 343, edited by M. Month and S. Turner (Springer-Verlag, 1989).

F. Neri and G. Rangarajan, Kinematic Moment Invariants for Linear Hamiltonian Systems, Phys. Rev. Lett. 64, p. 1073 (1990).

A.J. Dragt et al., General Moment Invariants for Linear Hamiltonian Systems, Physical Review A, 45 (1992).

Y. Yan, A.J. Dragt, et al., Comment on Round-Off Errors and on One-Turn Taylor Maps, Nonlinear Problems in Future Particle Accelerators, W. Scandale and G. Turchetti, Ed., World Scientific (1991).

A.J. Dragt, I.M. Gjaja, and G. Rangarajan, Kick Factorization of Symplectic Maps, Proceedings of the IEEE Accelerator Conference, p. 1621 (1991).

A.J. Dragt, G. Rangarajan, and F. Neri, Invariant Metrics for Hamiltonian Systems, Proceedings of the IEEE Accelerator Conference, p. 1630 (1991).

G. Rangarajan and F. Neri, Canonical Representations of $\mathrm{Sp}(2 \mathrm{n}, \mathrm{R})$, J. Math. Phys. 33, p. 1247 (1992).

I. Gjaja, Exact Evaluation of Arbitrary Symplectic Maps (1992).

1. Gjaja and A. Bhattacharjee, Effective Density Matrix for Free Electron Laser Radiation, Phys. Rev. A, 43 (1991). 
I. Gjaja and A. Bhattacharjee, Asymptotics of Reflectionless Potentials (1992).

J. van Zeijts and F. Neri, The Arbitrary Order Design Code Tlie 1.0 (1992).

A.J. Dragt et al., MARYLIE 3.0 User's Manual, A Program for Charged Particle Beam Transport Based on Lie Algebraic Methods (1991).

A.J. Dragt, Review of the SLAC Final Focus Test Beam Facility (1990).

A.J. Dragt, Lectures on Nonlinear Dynamics And Lie Methods With Applications to Accelerator Physics (225 pp.) (1991).

G. Rangarajan, Invariants for Symmplectic Maps and Symplectic Completion of Symplectic Jets, U. of Maryland Ph.D. thesis (1990).

\subsection{Task B}

R.L. Gluckstern and F. Neri, Beam Breakup with Coupling Between Cavities, Particle Accelerators, 모, 11 (1989).

F. Neri and R.L. Gluckstern, Single Bunch Cumulative Beam Breakup, Proceeding of the Particle Accelerator Conference, Chicago, IL, March 1989, p. 812.

R.L. Gluckstern, F. Neri and J.B.J van Zeijts, Suppression of Single Bunch Beam Breakup by BNS Damping, Proceedings of the Linac Conference, Albuquerque, NM (1990), p. 309.

R.L. Gluckstern and J.B.J. van Zeijts, Suppression of Single Bunch Beam Breakup by Autophasing, Proceedings of the Particle Accelerator Conference, San Francisco, CA (1991), p. 452.

R.L. Gluckstern, High Frequency Behavior of the Longitudinal Impedance for a Cavity of General Shape, Phys. Rev. D, 39, 2773 (1989). 
R.L. Gluckstern, Longitudinal Impedance of a Periodic Structure at High Frequency, Phys. Rev. D, $\underline{39}, 2780$ (1989).

R.L. Gluckstern and F. Neri, Longitudinal Coupling Impedance of a Small Obstacle, Proceedings of the Particle Accelerator Conference, Chicago, IL, March 1989, p. 1271.

R.L. Gluckstern, High Frequency Dependence of the Coupling Impedance for a Large Number of Obstacles, Proceedings of the Particle Accelerator Conference, Chicago, IL, March 1989, p. 1157.

R.L. Gluckstern and R. Li, Analysis of Coaxial Wire Measurement of Longitudinal Coupling Impedance, Particle Accelerators, 29, 159 (1990).

I. Gjaja, R. Li and R.L. Gluckstern, Impedance for a Multi-Cell, Multi-Block Structure, Proceedings of the Particle Accelerator Conference, San Francisco, CA, May 1991, p. 452.

R.L. Gluckstern, Longiturinal Coupling Impedance of an Iris in a Beam Pipe, CERN Report SL/90-113 (AP) (1990).

R.L. Gluckstern and W.F. Detlefs, Longitudinal Coupling Impedance of a Thin Iris Collimator, Proceedings of the Particle Accelerator Conference, San Francisco, CA, May 1991, p. 1600.

R.L. Gluckstern, R. Li and R.K. Cooper, Electric Polarizability and Magnetic Susceptibility of Small Holes in a Thin Screen, IEEE Transactions in Microwave Theory and Techniques Vol. 38, No. 2, 186 (1990); see also Vol. 38 , No. 10 (1990), pp.1529-1530.

R.L. Gluckstern and J.A. Diamond, Penetration of Fields through a Circular Hole in a Wall of Finite Thickness, IEEE Transactions in Microwave Theory and Techniques, Vol. 39, No. 2, 274 (1991). 
L.H. Yu, S. Krinsky and R.L. Gluckstern, Calculation of a Universal Scaling Function for Free Electron Laser Gain, Phys. Rev. Letters $\underline{64}, 3011$ (1990).

R.L. Gluckstern, Orbit Dynamics in the Field of a Bifilar Helix, LANL Report AT-6:ATN-89-20 (1989).

R.L. Gluckstern, Effects of Fringe Fields and Errors in a Bifilar Helix, LANL Report AT-6:ATN-89-21 (1989).

R.L. Gluckstern, Slotted Cylindrical Shell for Use as a Compact Wiggler, Phys. Rev. A15, 44, 3889 (1991).

L.H. Yu, S. Krinsky, R.L. Gluckstern and J.B.J. van Zeijts, The Effects of Wiggler Errors on FEL Gain, Phys. Rev. A15, 노, 1163 (1992).

R.L. Gluckstern, Coupling Impedance of a Single Hole in a Thick Wall Beam Pipe, CERN Report SL/92-05 (AP) (1992).

R.L. Gluckstern, Coupling Impedance of Many Holes in a Liner within a Beam Pipe, CERN Report SL/92-05 (AP) (1992).

R.L. Gluckstern, Analytic Field Model of an RFQ with Radial Vanes, LANL Report, AT-6-ATN-89-3 (1989).

R.L. Gluckstern, Beam Loading in an RFQ, LANL Report AT-6:ATN-89-4 (1989).

R.L. Gluckstern, External Q for a Cavity Radiating into a Waveguide, LANL Report AT-6:ATN-89-9.

R.L. Gluckstern, Wakefields in a Dielectric Wave Guide, LANL Report AT6:ATN-90-1 (1990).

R.L. Gluckstern, Thermal Distribution on a Slotted Cylindrical Shell, LANL Report AT-6:ATN-90-7 (1990). 
R.L. Gluckstern, Analysis of a Possible Beam Position Monitor for the Next Linear Collider, LANL, Report AT-6:ATN-90-13 (1990).

R.L. Gluckstern, Completeness of the Cavity Kernel Used in Calculating Longitudinal Coupling Impedance, CERN R:port SL/90-99 (AP) (1990).

R.L. Gluckstern, Compensation of Single Bunch Transverse Beam Breakup in a Chain of Long Periodic Linac Cavities, CERN Report SL/90-? (AP) (1990).

R.L. Gluckstern, RF Coupling, Accelerator Summer School Lectures, BNL, July 1989.

R. Li, The Analytical and Numerical Investigation of the Longitudinal Coupling Impedance, U. of Maryland, Ph.D. thesis (1990).

Keprints + Preprint remored. 

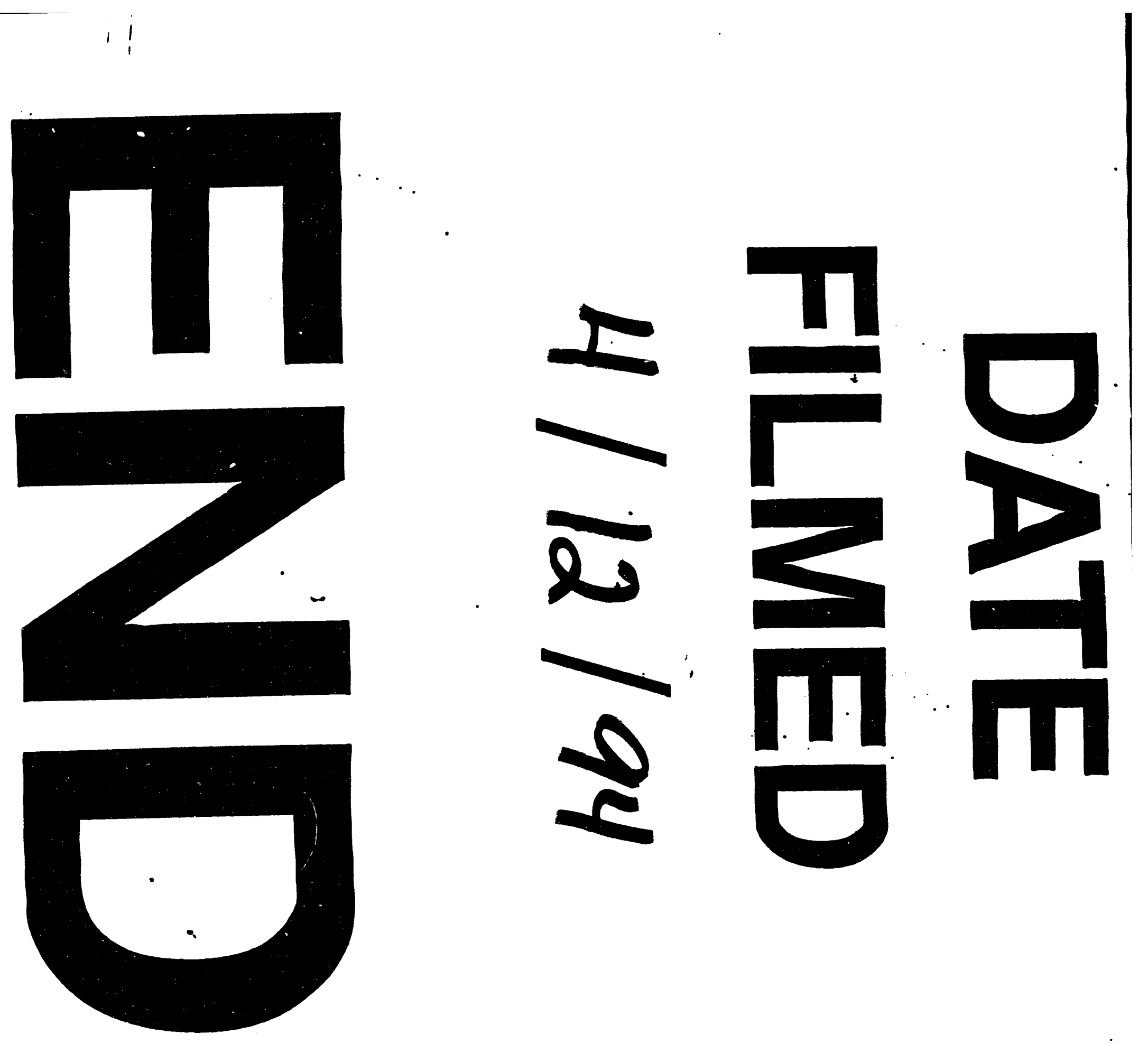
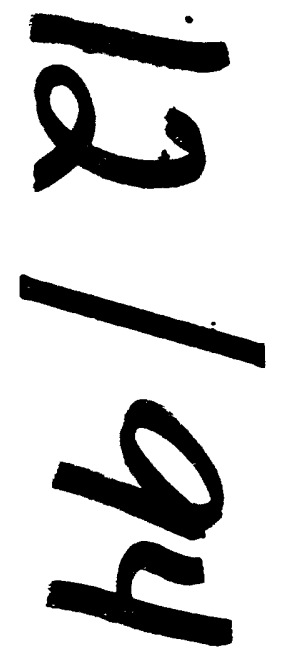


$$
-
$$
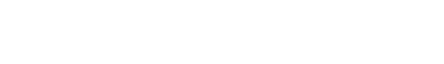